\title{
28 Research Soure \\ The clinical spectrum of COVID-19 in neonates and infants: A systematic review protocol
}

Ahreen Allana ( $\nabla$ ahreen_allana@hotmail.com )

Aga Khan University https://orcid.org/0000-0001-7918-142X

Sumera Aziz Ali

Columbia University

Ali Faisal Saleem

Aga Khan University

\section{Protocol}

Keywords: COVID-19, coronavirus, severe acute respiratory syndrome coronavirus, neonates, infants, pregnancy, vertical transmission

Posted Date: May 29th, 2020

DOI: https://doi.org/10.21203/rs.3.rs-31229/v1

License: @ (i) This work is licensed under a Creative Commons Attribution 4.0 International License. Read Full License 


\section{Abstract}

\section{Background}

Preliminary data on COVID-19 in the pediatric population has revealed that children account for a milder clinical course and better prognosis compared with adults. However, infants are at the greatest risk within this subset, with studies demonstrating the highest proportion of critical cases in this age group. The clinical spectrum of the disease in these children remains largely unexplored. Similarly, there is a dearth of research on the potential vertical/perinatal transmission of SARS-nCoV and the clinical outcomes of infants born to mothers who have been tested positive for the virus. In this review, we aim to summarize available literature on the clinical spectrum of COVID-19 in neonates and infants.

\section{Methodology}

This review will explore the clinical characteristics of COVID-19 in infants tested positive for SARS-nCoV using RT-PCR assays as well as neonates born to mothers who were detected with the virus during pregnancy. We will conduct a comprehensive search of two large databases, i.e. PubMed and Google Scholar and shortlist published and unpublished (pre-proof and pre-proof accepted) articles in English between 1 st November 2019 and 15 th May 2020 that meet the eligibility criteria. Keeping in view the paucity of data on the study topic, we plan to include all relevant articles irrespective of study design. The findings will be synthesized narratively following an analytical interpretation to capture emerging themes.

\section{Discussion}

Through this systematic review, we hope to provide an evidence-based framework for pediatricians to incorporate into their practice while managing neonates and infants tested positive for COVID-19 or those born to mothers who tested positive for the disease. This will set the stage for prospective clinical research to address the questions that remain unanswered. More importantly, exploring disease pathogenesis and transmission in pediatric patients could provide practical insights to guide future therapeutic and preventative measures and, perhaps, vaccine development.

Systematic review registration: This review has been submitted for registration with PROSPERO (acknowledgement of receipt -185333).

\section{Background}

The novel coronavirus (SARS-CoV-2) belongs to the family of coronaviridae and has been identified to be the causative pathogen for the coronavirus disease 2019 (COVID-2019) (1,2). Ever since the first case of 2019-nCoV was detected in Wuhan in the Hubei district of China in late November 2019, the virus has spread rapidly (3), owing to its highly contagious nature, across countries and continents $(4,5)$. On $11^{\text {th }}$ March 2020, the WHO officially declared the COVID-19 to be a pandemic which had then affected 
168,000 individuals and claimed 6500 lives across the globe (6). An interactive web-based dashboard set up by the Center for Systems Science and Engineering (CSSE) at Johns Hopkins University, Maryland, USA estimates the total number of cases to be 3,939,119 which includes 1,322,289 recoveries and 274,917 fatalities as of May 9 th $^{\text {th }}, 2020$ (7).

Preliminary data on COVID-19 in the pediatric population has revealed a prevalence of $1-5 \%$ (8), with children accounting for a milder clinical course and better prognosis compared with adults $(9,10)$. Despite their weaker immune defenses and a higher vulnerability to infectious processes, the mortality rate in children also appears to be significantly low (9). The theories being postulated for this protective effect of age include variations in pediatric immune responses and airway epithelial cell receptors that mediate viral binding (11). While these statistics can be reassuring to the pediatrician, the data could be misleading as many infected children have been found to be asymptomatic carriers $(9,12)$. Therefore, the role of this population in the community transmission of the disease should not be underestimated, especially with studies demonstrating both nasal and fecal viral shedding for several weeks following the initial diagnosis (13).

Despite the milder disease course of SARS-CoV-2 amongst children, infants under 1 year of age remain the greatest at risk within this subset. They are also the most likely to develop serious complications of the viral illness necessitating hospitalization and often critical care. Data from the United States has revealed the highest rate of pediatric hospitalizations in children aged $<1$ year between $12^{\text {th }}$ February and $2^{\text {nd }}$ April 2020 who accounted for $15 \%-62 \%$ of hospitalizations among pediatric patients with COVID19. Out of these, $62 \%$ required ICU level management (14). Similarly, in a study on Chinese pediatric patients diagnosed with SARS-CoV-2, Dong et. al noted the highest proportion of severe and critical cases $(10.6 \%)$ amongst infants less than 1 year compared with $7.3 \%, 4.2 \%, 4.1 \%$, and $3.0 \%$ for the age groups $1-5,6-10,11-15$ and $>15$ years respectively (15).

The primary mode of transmission of the virus has been reported to be person to person $(15,16)$ through respiratory droplets and close contact but studies have also postulated oro-fecal transmission as a potential route $(17,18)$. The limited data on vertical transmission has shown a low risk of maternal to fetal transmission (19-22). However, reports from both China and elsewhere have shown positive results in infants born to mothers who were clinically suspected or diagnosed to be COVID-19 positive (23-25). Whether this was a result of placental or antepartum transmission or occurred following delivery through aerosol infection remains questionable. Additionally, the symptoms and clinical manifestations in SARSCoV-2 negative newborns born to infected mothers are variable and non-specific, ranging from no symptoms to fever and respiratory distress $(26,27)$.

One may question if this diverse clinical presentation could be attributed to vertical transmission (23) of the virus or the transfer of protective antibodies transplacentally or through breast milk (28), that could, perhaps, account for the relatively milder disease course in newborn infants. However, these entities remain largely unexplored and require extensive research to review both existing data and set recommendations for future trials. The recent UNICEF estimate of 116 million babies to be born globally 
in the wake of the pandemic is worrisome and could potentially place an enormous burden on the healthcare system (29). It, therefore, becomes, even more essential to study the clinical spectrum of the disease in newborns and explore the possibility of vertical transmission.

In this review, we aim to summarize available literature on the clinical spectrum of COVID-19 in neonates and infants who have been tested positive for the disease using RT-PCR assays. The primary objective is to shed light on the clinical manifestations, diagnostic modalities, prognosis, management options and preventive strategies that have been employed by pediatricians across the globe to curb the spread of this infection in this population subset. Our secondary objective is to explore in greater detail the incidence of maternal transmission and clinical manifestations in newborns of COVID-19 positive mothers. This review will analyze what is known already about the clinical manifestations of the disease in neonates and infants and management strategies employed by neonatologists and pediatricians to treat both infants tested positive for the disease and those born to COVID-19 positive mothers. It will also obtain information on potential vertical/perinatal transmission and neonatal outcomes in pregnant women infected with SARS-CoV-2.

Through this systematic review, we, therefore, hope to provide an evidence-based framework for pediatricians to incorporate into their practice while managing these infants and set the stage for prospective clinical research to address the questions that remain unanswered. More importantly, exploring disease pathogenesis and transmission in pediatric patients could possibly provide practical insights to guide future therapeutic measures and vaccine development.

This study has two key objectives:

- To outline the clinical manifestations, laboratory and imaging findings, management options and prevention strategies in neonates and infants tested positive for SARS-CoV-2 using RT-PCR based assays.

- To summarize existing data on vertical/perinatal transmission, and clinical characteristics and outcomes of neonates born to pregnant women infected with SARS-CoV-2.

\section{Material And Methods}

This review will focus on the clinical spectrum of COVID-19 in neonates and infants who have been tested positive for the disease using RT-PCR assays, and newborns born to mothers who were tested positive for the viral infection using RT-PCR asaays. This will allow pediatricians to manage these infants and set recommendations for prospective clinical research to address additional questions.

\section{Eligibility Criteria}

Table 1 summarizes the inclusion and exclusion criteria that will be used to shortlist studies for the review. In summary, we will include published and unpublished (pre-proof or pre-proof accepted) articles on pregnant women, neonates and infants tested positive for COVID-19 using RT-PCR assays. Articles 
that have either been published or approved for publication in indexed journals will be included. Keeping in view the paucity of data on the study topic, we plan to include all relevant articles irrespective of study design. This would include case reports, case series, cross-sectional studies and clinical trials among others. Since the first case of the disease was reported in late November 2019, we will limit our search to studies published or accepted for publication between November 2019 to May $15^{\text {th }}, 2020$. Studies in languages other than English will be excluded.

\section{Information sources and search strategy}

An electronic systematic literature search will be carried out to outline the clinical manifestations, laboratory and imaging findings and management options in neonates and infants tested positive for SARS-CoV-2 using RT-PCR based assays and to summarize existing data on vertical/perinatal transmission and neonatal characteristics and outcomes in pregnant women infected with SARS-CoV-2. Although there are many databases with relevant articles on the topic of interest, we will search two large electronic databases i.e. PubMed and Google Scholar for the current systematic review. These databases will be searched independently by two researchers who will use a detailed search strategy comprising of search terms or combinations. The search strategy will first be piloted to ensure adequate specificity and sensitivity. Articles will then be searched using a combination of search terms defined to address the research questions. The preliminary search strategy is illustrated in Table 2 in which search items have been grouped according to the PICOS criteria: 1) Population (infants less than 1 year or pregnant women), 2) Intervention /Exposure (tested positive for SARS-CoV-2 using PCR based assays), 3) Comparison group (not applicable), 4) Outcome (clinical manifestations, diagnosis, management, prognosis and prevention), and 5) Settings (developing and developed countries). The pertinent search terms will be put together using combinations (AND, OR) germane to the research question. Moreover, we will use truncation $(*)$ to identify additional research articles with the same root word. Additionally, indexed keywords in the Medical Subject Headings (MeSH) will be used to ensure uniform search terms. The MeSH terms, 'Coronavirus*', 'COVID', 'COVID-19', 'neonate*', 'infant less than 28 weeks', 'infant*', 'infant less than 1 year' will be used. We will also apply search limits or filters on publication year (20192020), language (English), age group (neonates and infants less than 1 year) and type of studies (quantitative observational) to include eligible articles in the search.

\section{Study selection}

Citation management system (Endnote software) will be used to manage the records exported from all the electronic databases. In the first step, all studies will be screened by study titles using the Endnote software. The shortlisted studies will then be screened by reviewing study abstracts. Lastly, the full text of selected studies will be retrieved and screened against the eligibility criteria. In order to ensure the reliability of shortlisting articles between the two reviewers, a pre-defined screening form will be developed and piloted as per the eligibility criteria. Both reviewers will describe outcome measures after reviewing the studies to verify the relevance of the articles. Strong justifications for excluding studies will 
be provided by each reviewer. Any disagreement between the two reviewers will be resolved by a third reviewer in a consensus meeting. The third reviewer will be consulted to make the final decision about whether the study meets the eligibility criteria for inclusion. The PRISMA flow diagram (Figure 1) will be used to report the study selection process.

\section{Data collection process}

A customized data extraction sheet will be filled by two independent reviewers for the eligible studies. Data extraction tables of both reviewers will be matched to ensure that all key findings are included in the systematic review. A third evaluator will be involved, if discordant information is observed during the data extraction process. The data extraction sheet will be pilot tested before initiating the data extraction process. Alongside, existing studies on this research area have been reviewed to determine items for the data extraction form. The items included in the preliminary data extraction form include the title of the article, author, publication date, country of study, date of extraction, reviewer name, study type, study population, relevant findings, and study limitations.

\section{Quality assessment of eligible studies}

Both reviewers will independently assess the quality of each study using different Newcastle-Ottawa Scales for cross-sectional and cohort studies. This scale is used to assess the quality of descriptive and analytical studies by assessing potential sources of bias in the selection, comparability of participants and the assessment of study outcome. This scale has a maximum of 4 points for selection, 2 for comparability and 3 for outcome, leading to a total maximum score of 9 . Each eligible study will be scored against 9 maximum points with studies acquiring higher scores depicting higher quality.

\section{Synthesis of included studies}

The findings of the review will first be synthesized narratively. Initially, we will perform a descriptive analysis of all the final included studies to record their main characteristics such as study title, authors, publication year, study aim, study methods, sampling strategy, and characteristics of study participants. To begin with, two independent reviewers will read each included study article multiple times to extract data and individual group-related results. Following this, the reviewers will record analytical interpretations of findings to capture emerging themes. Finally, the findings will be presented in the form of a narrative review as well as interactive tables and charts to summarize key findings.

\section{Discussion}

Data on the clinical manifestations, prevention and management of COVID-19 is rapidly evolving. With the limited data available on the clinical spectrum of the disease in the pediatric population, it is apparent that infants less than 1 year of age are typically the ones to be most severely affected, often warranting 
high level critical care. In addition to this, there is a dearth of research on the potential vertical/perinatal transmission of SARS-CoV-2. This is a matter of significant concern as it poses a challenge to obstetricians and neonatologists regarding the preventative measures that they must adopt to decrease the likelihood of transmission and the diagnostic and treatment modalities that they must resort to should the newborn be tested positive for the novel coronavirus.

This protocol will lead to a systematic review that will summarize available data on the clinical course of the disease in newborns and infants. By doing so, we hope to shed light on what is being done currently in different parts of the world to manage the disease in neonates and infants. It will also provide an insight into the possibility of vertical transmission of the virus from studies on pregnant women that have been reported so far.

The review will put forward an evidence-based framework that can be used in the clinical setting to manage these infants. Since existing reports have identified most children tested positive for COVID-19 to present with a milder disease course or be asymptomatic carriers (30), curtailing the spread of the disease amongst the pediatric population could potentially limit transmission to adults and the elderly, who have been shown to have more severe symptoms with possible fatal consequences (31).

Knowledge about the clinical manifestations of COVID-19 in neonates and infants as well as diagnostic, preventive and treatment options being used to manage them will also help guide effective therapeutic and preventative measures, including, perhaps the prospects of vaccine development targeted at curbing the spread of the infection amongst this highly vulnerable pediatric age group.

\section{Declarations}

\section{Consent for publication}

Not applicable

\section{Availability of data and materials}

Materials described in this paper pertain to the protocol only and there are no raw data reported. The studies will be retrieved and analyzed and can be made available from the corresponding author upon reasonable request.

\section{Competing interests}

The authors declare that they have no competing interests.

\section{Funding}

None 
The study was conceptualized by AA. AA prepared the first draft of the manuscript and SAA and AFA edited the draft and supervised AA to develop the protocol and write different sections of the protocol. All authors have contributed to this manuscript and reviewed and approved the final version of the paper.

\section{Acknowledgements}

None

\section{References}

1.Chen Y, Liu Q, Guo D. Emerging coronaviruses: Genome structure, replication, and pathogenesis. J Med Virol. 2020;92(4):418-23.

2.Tu H, Tu S, Gao S, Shao A, Sheng J. The epidemiological and clinical features of COVID-19 and lessons from this global infectious public health event. J Infect. 2020.

3.Liu SL, Saif L. Emerging Viruses without Borders: The Wuhan Coronavirus. Viruses. 2020;12(2).

4.Nishiura H, Jung SM, Linton NM, Kinoshita R, Yang Y, Hayashi K, et al. The Extent of Transmission of Novel Coronavirus in Wuhan, China, 2020. J Clin Med. 2020;9(2).

5.Khachfe HH, Chahrour M, Sammouri J, Salhab H, Makki BE, Fares M. An Epidemiological Study on COVID-19: A Rapidly Spreading Disease. Cureus. 2020;12(3):e7313.

6.Mahase E. Covid-19: WHO declares pandemic because of "alarming levels" of spread, severity, and inaction. Bmj. 2020;368:m1036.

7.Dong E, Du H, Gardner L. An interactive web-based dashboard to track COVID-19 in real time. Lancet Infect Dis. 2020.

8.Ludvigsson JF. Systematic review of COVID-19 in children show milder cases and a better prognosis than adults. Acta Paediatrica. 2020.

9.de Souza TH, Nadal JA, Nogueira RJN, Pereira RM, Brandao MB. Clinical Manifestations of Children with COVID-19: a Systematic Review. medRxiv. 2020.

10.Hong H, Wang Y, Chung HT, Chen CJ. Clinical characteristics of novel coronavirus disease 2019 (COVID-19) in newborns, infants and children. Pediatr Neonatol. 2020;61(2):131-2.

11.Yonker LM, Shen K, Kinane TB. Lessons unfolding from pediatric cases of COVID-19 disease caused by SARS-CoV-2 infection. Pediatr Pulmonol. 2020;55(5):1085-6.

12.Hu Z, Song C, Xu C, Jin G, Chen Y, Xu X, et al. Clinical characteristics of 24 asymptomatic infections with COVID-19 screened among close contacts in Nanjing, China. Sci China Life Sci. 2020;63(5):706-11. 
13.Zhang W, Du RH, Li B, Zheng XS, Yang XL, Hu B, et al. Molecular and serological investigation of 2019nCoV infected patients: implication of multiple shedding routes. Emerg Microbes Infect. 2020;9(1):386-9.

14.Coronavirus Disease 2019 in Children - United States, February 12-April 2, 2020. MMWR Morb Mortal Wkly Rep. 2020;69(14):422-6.

15.Dong Y, Mo X, Hu Y, Qi X, Jiang F, Jiang Z, et al. Epidemiology of COVID-19 Among Children in China. Pediatrics. 2020.

16.Li Q, Guan X, Wu P, Wang X, Zhou L, Tong Y, et al. Early Transmission Dynamics in Wuhan, China, of Novel Coronavirus-Infected Pneumonia. N Engl J Med. 2020;382(13):1199-207.

17.Gu J, Han B, Wang J. COVID-19: Gastrointestinal Manifestations and Potential Fecal-Oral Transmission. Gastroenterology. 2020.

18.Tian Y, Rong L, Nian W, He Y. Review article: gastrointestinal features in COVID-19 and the possibility of faecal transmission. Aliment Pharmacol Ther. 2020;51(9):843-51.

19.Della Gatta AN, Rizzo R, Pilu G, Simonazzi G. COVID19 during pregnancy: a systematic review of reported cases. American Journal of Obstetrics \& Gynecology. 2020.

20.Parazzini F, Bortolus R, Mauri PA, Favilli A, Gerli S, Ferrazzi E. Delivery in pregnant women infected with SARS-CoV-2: A fast review.

Outcome of Coronavirus spectrum infections (SARS, MERS, COVID 1-19) during pregnancy: a systematic review and meta-analysis. American Journal of Obstetrics \& Gynecology MFM. 2020:100107.

22.Chen H, Guo J, Wang C, Luo F, Yu X, Zhang W, et al. Clinical characteristics and intrauterine vertical transmission potential of COVID-19 infection in nine pregnant women: a retrospective review of medical records. The Lancet. 2020;395(10226):809-15.

23.Alzamora MC, Paredes T, Caceres D, Webb CM, Valdez LM, La Rosa M. Severe COVID-19 during Pregnancy and Possible Vertical Transmission. Am J Perinatol. 2020.

24.Yu N, Li W, Kang Q, Xiong Z, Wang S, Lin X, et al. Clinical features and obstetric and neonatal outcomes of pregnant patients with COVID-19 in Wuhan, China: a retrospective, single-centre, descriptive study. Lancet Infect Dis. 2020.

25.Zeng L, Xia S, Yuan W, Yan K, Xiao F, Shao J, et al. Neonatal Early-Onset Infection With SARS-CoV-2 in 33 Neonates Born to Mothers With COVID-19 in Wuhan, China. JAMA Pediatr. 2020.

26.Yang P, Wang X, Liu P, Wei C, He B, Zheng J, et al. Clinical characteristics and risk assessment of newborns born to mothers with COVID-19. J Clin Virol. 2020;127:104356. 
27.Zhu H, Wang L, Fang C, Peng S, Zhang L, Chang G, et al. Clinical analysis of 10 neonates born to mothers with 2019-nCoV pneumonia. TransI Pediatr. 2020;9(1):51-60.

28.Zeng H, Xu C, Fan J, Tang Y, Deng Q, Zhang W, et al. Antibodies in Infants Born to Mothers With COVID-19 Pneumonia. Jama. 2020.

29.Pregnant mothers and babies born during COVID-19 pandemic threatened by strained health systems and disruptions in services [press release]. UNICEF, 7th May 20202020.

30.Kelvin AA, Halperin S. COVID-19 in children: the link in the transmission chain. Lancet Infect Dis. 2020.

31.Verity R, Okell LC, Dorigatti I, Winskill P, Whittaker C, Imai N, et al. Estimates of the severity of coronavirus disease 2019: a model-based analysis. Lancet Infect Dis. 2020.

\section{Tables}

\begin{tabular}{|l|l|l|}
\hline \multicolumn{1}{|c|}{ Table 1: Eligibility Criteria defined to include relevant articles } \\
\hline $\begin{array}{l}\text { Attribute } \\
\text { Population }\end{array}$ & $\begin{array}{l}\text { Inclusion Criteria } \\
\text { tested positive for COVID-19 } \\
\text { Infants aged 1 month to 1 year tested } \\
\text { positive for COVID-19 } \\
\begin{array}{l}\text { Pregnant women tested positive for } \\
\text { COVID-19 where neonatal outcomes } \\
\text { and clinical characteristics (including } \\
\text { laboratory and/or imaging findings) } \\
\text { have been reported. }\end{array}\end{array}$ & $\begin{array}{l}\text { Exclusion Criteria } \\
\text { Children aged more than 1 year } \\
\text { conditions } \\
\text { Studies on pregnant women } \\
\text { tested positive for COVID-19 } \\
\text { where neonatal clinical } \\
\text { characteristics and neonatal } \\
\text { outcomes have not been } \\
\text { reported. }\end{array}$ \\
\hline $\begin{array}{l}\text { Study } \\
\text { design }\end{array}$ & $\begin{array}{l}\text { All observational and interventional } \\
\text { studies including case series, case } \\
\text { reports, cross sectional studies clinical } \\
\text { trials }\end{array}$ & $\begin{array}{l}\text { Reviews including systematic } \\
\text { reviews and meta-analysis }\end{array}$ \\
\hline Language & $\begin{array}{l}\text { Studies published or pre-published in } \\
\text { English }\end{array}$ & $\begin{array}{l}\text { Studies where translation in } \\
\text { English is not available }\end{array}$ \\
\hline $\begin{array}{l}\text { Time } \\
\text { duration }\end{array}$ & $\begin{array}{l}\text { st Nov 2019 to May 15 } \\
\text { th 2020 }\end{array}$ & $\begin{array}{l}\text { Studies published after May 15 } \\
\text { 2020 }\end{array}$ \\
\hline Journals & $\begin{array}{l}\text { Studies that are freely accessible and } \\
\text { published or accepted for publication } \\
\text { in indexed journals }\end{array}$ & $\begin{array}{l}\text { Studies not published or accepted } \\
\text { for publication }\end{array}$ \\
\hline
\end{tabular}




\section{le 2: Search strategy using PICO framework}

\begin{tabular}{l|l|}
\hline ulation & $\begin{array}{l}\text { Neonate*[MesH] OR Newborn* [MesH] OR infant* [MeSH] OR } \\
\text { infant less than 1 month[MeSH] OR infant less than 28 } \\
\text { weeks[MeSH] OR pregnant women [MeSH]. }\end{array}$ \\
\hline rvention/exposure & $\begin{array}{l}\text { COVID-19 OR COVID OR coronavirus* OR SARS-CoV-2 [MeSH } \\
\text { Terms]. }\end{array}$ \\
\hline aparison group & Not Applicable \\
\hline come & $\begin{array}{l}\text { Clinical* OR symptoms OR signs OR prognosis OR mortality OR } \\
\text { diagnosis OR imaging OR laboratory* OR management OR } \\
\text { treatment OR prevention. }\end{array}$ \\
\hline $\begin{array}{l}\text { Developing and developed counties. } \\
\text { כrs }\end{array}$ & $\begin{array}{l}\text { Published or unpublished (pre-proof accepted) between 1st } \\
\text { November 2019 and 15 }\end{array}$ \\
\hline
\end{tabular}

